\title{
MOMENTUM PROJECT: BUSCANDO EL EQUILIBRIO ENTRE LA CREACIÓN DE VALOR ECONÓMICO Y VALOR SOCIAL
}

\author{
Autores: Luisa Alemany Gil ${ }^{1}$ \\ Profesora titular \\ ESADE Business School, URL \\ Alfred Vernis Domènech \\ Profesor titular \\ ESADE Business School, URL
}

\section{Resumen}

El presente artículo analiza un caso de estudio sobre inversión de impacto en España. Momentum Project es una iniciativa que surge para apoyar a los emprendedores sociales a escalar su empresa, con el objeto de incrementar el retorno

\footnotetext{
${ }^{1}$ Contacto autores: Luisa Alemany Gil: luisa.alemany@esade.edu y Alfred Vernis Domènech: alfred. vernis@esade.edu

Los autores quieren agradecer al BBVA su ayuda, apoyo y disponibilidad durante las entrevistas y en la revisión de este artículo. En especial queremos dar las gracias a A. Ballabriga, L. del Pozo, A. Calvo, J.Guinea, E. Migoya y A. Sánchez, sin ellos este artículo no habría sido posible.
} 
social y conseguir la sostenibilidad a largo plazo de las empresas. En un primer momento se plantea como una "aceleradora" para emprendedores sociales, sin embargo, se acaba implementando un vehículo de inversión de impacto para poder dar apoyo a las necesidades financieras del plan de crecimiento. Las características del vehículo de Momentum son comparadas con las del capital riesgo filantrópico de acuerdo a la literatura académica.

Palabras clave: emprendeduría social; inversión de impacto; capital riesgo filantrópico;

Momentum project: in search for equilibrium between social and economic value

\begin{abstract}
This paper analyzes an in-depth case analysis on impact investment in Spain. Momentum Project is an initiative to support social entrepreneurs in their path to growth. The goal is to increase the social return and to help the social enterprises to reach their long-term sustainability. At the beginning, Momentum started as an "accelerator" for social entrepreneurs; however, an impact investment vehicle is created to support the financial needs of the growth plan. The characteristics of the investment vehicle are compared to those of the philanthropic venture capital as studied in the academic literature.
\end{abstract}

Key words: social entrepreneurship; impact investing; philanthropic venture capital

\title{
1. INTRODUCCIÓN
}

Durante la última década el capital riesgo filantrópico, también conocido como inversión de impacto, se ha desarrollado mucho, tanto en Estados Unidos como en Europa. Este nuevo modelo de financiación para las empresas sociales pasa de la donación o subvención a fondo perdido a una inversión en fondos propios o deuda, donde el aportante de fondos no se comporta como un donante si no como un inversor de capital riesgo. La similitud con el capital riesgo reside en el valor añadido y el seguimiento de la empresa participada. La gran diferencia con el capital riesgo es que no se busca la maximización del beneficio para el inversor, si no la maximización del impacto social. 
El objetivo del modelo de venture philanthropy es que la empresa social financiada consiga ser sostenible por si misma, es decir, alcance el punto de equilibrio que le permita crecer y llegar a un mayor número de personas, maximizando su impacto social (Letts, Ryan \& Grossman, 1997).

Desde el punto de vista del financiador, se trata de un cambio de mentalidad, pasando de hacer donaciones por proyecto (lógica de la filantropía tradicional) a invertir en empresas (economía de mercado), es decir, que el enfoque económico financiero pasa a tener un papel clave, ya que la sostenibilidad de la empresa es la clave del éxito de la inversión. Es en este cambio donde se puede producir la tensión que es el objetivo de análisis de este artículo, ya que en la filantropía tradicional solo se espera obtener un retorno social, mientras que en la economía de mercado la rentabilidad financiera, aunque sea inferior, también es un objetivo.

Desde el punto de vista de la empresa social, el capital riesgo filantrópico representa una oportunidad, ya que además del dinero los inversores aportarán contactos, se convertirán en consejeros de la empresa, y ayudarán en aquellos aspectos clave para su futuro. La inversión es la necesaria para poder escalar la empresa y por lo tanto, el emprendedor social se puede enfocar en hacer crecer el negocio en lugar de tener que estar desviando su atención cada año a la búsqueda de nueva financiación, como sucede en el modelo de financiación tradicional de este tipo de empresas. Además, al ser el crecimiento un objetivo, se maximiza el retorno social.

El capital riesgo filantrópico integra la aportación de capital con el apoyo en temas de estrategia y gestión de empresas, lo que permite a la empresa social tener una visión emprendedora a largo plazo y competir en el mercado de manera independiente. La proposición de valor de esta forma de financiación de empresas sociales es el apoyo a empresas de alto impacto social con el objetivo de maximizar el "return on social investment" (Scarlata \& Alemany, 2008).

Durante el año 2013 se invirtió en el mundo alrededor de 11 mil millones de dólares en inversión de impacto en unas 5.000 operaciones. En el año 2014 se estima llegar a unos 13 mil millones de dólares en unas 6.500 operaciones $^{2}$. Aun así, este tipo de financiación para las empresas sociales todavía representa un nicho dentro del sector social, pero el crecimiento experimentado, así como el que cada día más fundaciones vean en la inversión de impacto la forma de conseguir de manera más eficiente su misión, parece indicar que en las próximas décadas este tipo de inversión pasará a ser común en el sector social.

El objetivo de este artículo es estudiar la tensión que se genera entre la creación de valor o impacto social y la rentabilidad económica esperada en una inversión. Para ello se analiza en profundidad Momentum Project, un programa

\footnotetext{
${ }^{2}$ Información estimada según la encuesta de la Global Impact Investing Network (GIIN) 2014. Para más información véase informe de JP Morgan, Mayo 2014.
} 
de apoyo y financiación a empresas sociales liderado por un banco, un centro universitario, y con el apoyo de una consultora. A partir del análisis de este caso se trata de entender el funcionamiento de la inversión de impacto en España. En el apartado 2 se repasa la literatura primero sobre el emprendimiento social y a continuación sobre el capital riesgo filantrópico. En el apartado 3, se describe la metodología. A continuación se presenta Momentum Project, primero su objetivo y su modo de actuación y luego el funcionamiento del vehículo de inversión de Momentum para apoyar a las empresas sociales. Finalmente, se presentan los resultados, las lecciones aprendidas y las conclusiones.

\section{REVISIÓN DE LA LITERATURA: EL EMPRENDIMIENTO SOCIAL Y EL CAPITAL RIESGO FILANTRÓPICO}

\subsection{El emprendimiento social}

Mientras que el término "emprendimiento social" es relativamente reciente, algunas de las actividades que han venido a ser asociadas con el término tienen sus inicios en la década de los setenta. En concreto, varios esfuerzos inconexos en países en vías de desarrollo se desplegaron durante esta década, como los préstamos de microcréditos. La ONG norteamericana Acción comienza a experimentar con soluciones de negocio para hacer frente a la pobreza a través de microcréditos alrededor del año 1973 en América Latina. Mientras que poco después, en 1976, el Banco Grameen de Bangladesh creado por el profesor Mohammad Yunus, empieza también a proporcionar microcréditos a los pobres. Estas organizaciones pioneras desarrollan enfoques distintos a la prestación de microcréditos a personas pobres, que al principio se constituyeron como una práctica marginal en el campo del desarrollo, y al cabo de los años han ocupado un espacio muy importante ${ }^{3}$. Es conveniente resaltar que el movimiento de los microcréditos viene de las ONG. Su evolución posterior ha provocado muchas tensiones entre el verdadero impacto social que generan en las personas que reciben los préstamos, y el valor económico que generan para las organizaciones de microcréditos y sus inversores.

Al mismo tiempo, Bill Drayton, después de haber trabajado como consultor, profesor universitario y directivo del gobierno, funda la organización internacional Ashoka. Una organización con el objetivo explícito de identificar innovadores de todo el mundo y apoyarlos con el fin de "acelerar el desarrollo". Es a Bill Drayton a quien se le atribuye la eventual acuñación del término "emprendedor social". Así,

\footnotetext{
${ }^{3}$ El último informe de la Microcredit Summit Campaign habla de 204 millones de clientes de las instituciones de microcrédito, y de estos, 116 millones de clientes entre los más pobres. Ver Larry R. Reed et al. (2014): Resilencia. El Informe del Estado de la Campaña de la Cumbre de Microcrédito 2014. Washington DC: Microcredit Summit Campaign.
} 
en los años 80, Ashoka se centra en una terminología sobre el término "emprendedor" y su fusión con "social", para indicar que el motivo de tal iniciativa empresarial era la promoción del bienestar social. (Ashoka, 2007). Como se puede ver, en este caso, el término "emprendedor social" se acuña en el sector empresarial.

En los últimos años, el emprendimiento social ha empezado a llamar poderosamente la atención del mundo académico. Leadbeater (1997), uno de los autores más citados por su trabajo seminal "The rise of social entrepreneurship", enfatizó en su obra la creciente necesidad de desarrollar de manera innovadora nuevas formas de capital social que fomenten la erradicación de la pobreza y la solución de los problemas sociales. Y, lo hizo revindicando la necesidad de que confluyeran aún más el sector público, el sector privado y el sector no lucrativo, y que de la combinación de los tres podrían salir emprendedores sociales que ayudaran a mejorar el Estado del Bienestar.

A pesar del creciente interés académico en el emprendimiento social, muchos autores destacan que falta una definición clara. Y, mantienen que esta labor se ha vuelto más compleja por la diversidad de tipos de emprendimientos sociales que se muestran y la variedad de comunidades académicas estudiando el tema (Mair y Martí, 2006; Peredo y McLean, 2006; Zahra et al., 2009).

Seguramente una de las personas que más ha ayudado a tratar de entender que es un emprendedor social ha sido Greg Dees. En 1998, en su artículo "The meaning of social entrepreneurship" 4 Dees analiza el origen de la palabra francesa "entrepreneurship" remontándose al economista francés del siglo XIX que escribió que "el emprendedor saca los recursos económicos de un área de poca productividad y los coloca en una de alta productividad y mayor rendimiento" (pp.114) Cita también la idea de Schumpeter del proceso "creativo-destructivo", para luego recuperar a Drucker y su concepto de "oportunidad". El emprendedor explota las oportunidades que el cambio crea. A partir de aquí Dees define a un emprendedor social como "un agente de cambio", y pide que se base el conocimiento sobre el emprendedor social en la teoría e investigación de "entrepreneurship". Dees trata pues de sacar la discusión de si el proyecto es social o económico, para centrarse en la idea de que lo importante es que sea "emprendimiento". Esta aproximación se acerca mucho al caso que se analiza en este artículo.

Frente a Dees otro grupo de investigadores se refiere al emprendimiento social como las iniciativas de las organizaciones no lucrativas que buscan nuevas formas de obtener recursos financieros (Emerson y Twersky, 1996; Norris, 1983; Skloot, 1983). Es verdad que con la crisis un número importante de organizaciones no lucrativas empezó a acercar al mercado sus programas principales, buscando así depender menos de las donaciones.

\footnotetext{
${ }^{4}$ Existe una traducción española del mismo publicada en la Revista Española del Tercer Sector, núm. 17, Enero-Abril 2011, pp. 111-121
} 
El propio Dees, junto con Anderson (2006) volvieron sobre el tema para resaltar que un emprendimiento social no es un tipo de organización no lucrativa. Lo que diferencia al emprendimiento social de una organización no lucrativa es la idea de utilizar métodos empresariales para crear nuevas maneras de alcanzar objetivos sociales. Dees y Anderson sugieren que lo que separa a la escuela del emprendimiento social del simple status de organización no lucrativa es la forma innovadora en la que se combinan métodos del mundo empresarial y del mundo no lucrativo para crear valor social.

En este debate, una de las grandes preocupaciones de diversos autores ha sido encontrar una definición de emprendimiento social. Entre los trabajos académicos centrados en definir este término (Bloom y Chatterji, 2009; Dees, 1998; Dees y Anderson, 2006; Mair y Marti, 2006; Martin y Osberg, 2007; Murphy y Combs, 2009; Peredo y McLean, 2006; Zahra et al, 2009) existe un amplio consenso en que un emprendimiento social implica la creación de valor social a través de medios innovadores y que a su vez contiene elementos inspirados en el mundo de los negocios.

Ponerse de acuerdo en una definición no es algo fácil. Pontikes (2012) y otros autores han tratado de trabajar sobre las clasificaciones ambiguas de las organizaciones en diferentes campos de la economía. Otro grupo de investigadores han preferido trabajar con la idea de un "continuum" entre las organizaciones no lucrativas y las empresas. Situándose las "empresas sociales" a la mitad de este "continuum" (ver figura 1 ).

Figura 1. El continuum nonprofit - forprofit

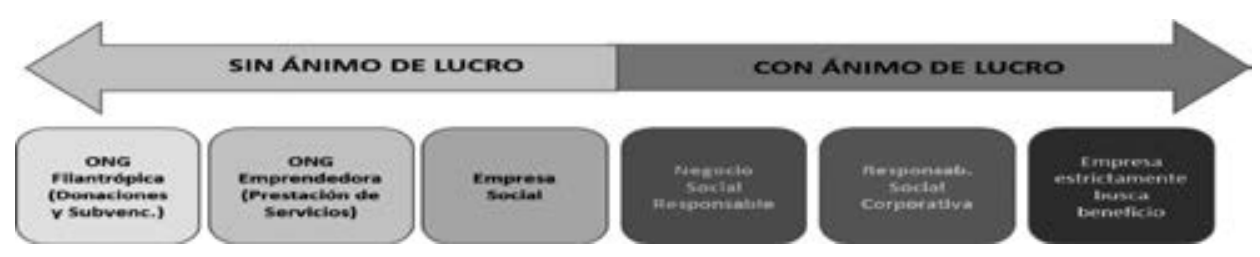

Fuente: Elaboración propia a partir de EVPA, 2010.

La idea del "continuum" ayuda a comprender otra de las denominaciones que últimamente se está utilizando para designar a las empresas sociales: "organizaciones híbridas". La combinación de aspectos empresariales y filantrópicos en la actividad central de una organización, genera unas dinámicas de gestión que trascienden las descritas por la literatura de gestión no lucrativa y de responsabilidad social corporativa (Battilana et al, 2012). Este continuum nos ayudará posteriormente para clasificar y entender la muestra de empresas y organizaciones que han participado hasta la fecha del Programa Momentum. 


\subsection{El capital riesgo filantrópico o inversión de impacto}

El concepto de capital riesgo filantrópico aparece por primera vez en 1997 (Letts et al., 1997), y se presenta como la aplicación de las técnicas de inversión del capital riesgo (Tyebjee y Bruno, 1984; Gompers y Lerner, 2001) en el mundo tradicional de las aportaciones filantrópicas. Sin embargo, esta definición limita el capital riesgo filantrópico a las fundaciones y ONGs, lo que no que no permitiría caracterizar otras instituciones dentro de esta categoría. Además, en esta primera definición no se determina cual es la propuesta de valor del capital riesgo, por lo que no queda claro quién es el receptor de los fondos.

La definición utilizada en este artículo es la de Scarlata y Alemany (2010), donde capital riesgo filantrópico "es una inversión en empresas sociales que tienen un alto potencial de impacto social. Debido a los problemas de información asimétrica entre los aportantes de fondos y los receptores de estos, el modelo de capital riesgo filantrópico proporciona capital y valor añadido, con el objetivo principal de maximizar el impacto social de la inversión” (pp. 123).

En el modelo de capital riesgo filantrópico los aportantes al fondo invierten con el objetivo de apoyar a empresas sociales con un alto potencial de impacto. En lugar de enfocarse en la maximización de la rentabilidad, como el capital riesgo tradicional, se busca maximizar el retorno social, aunque este retorno no es incompatible con el retorno financiero. La principal hipótesis en la que se apoya el modelo del capital riesgo filantrópico es que el impacto social se crea y se maximiza si la empresa social crece y alcanza el punto de equilibrio, siendo financieramente sostenible, pudiendo continuar su labor social en el largo plazo. Es en este punto donde puede existir tensión al tener dos objetivos, uno social y otro económico, que pueden parecer opuestos.

Para conseguir crecimiento y sostenibilidad, las dos palabras clave, el inversor de impacto aporta dinero y valor añadido, a través de su experiencia en la gestión de empresas, sus contactos, o cualquier otra aportación de valor. Los inversores de impacto invierten en un número limitado de empresas, dentro de una zona geográfica o en un sector específico, como salud, educación o pobreza. Se trata de añadir valor para asegurar que la empresa social cuente con todo el empuje de estos inversores de capital riesgo social. El tiempo de permanencia en la empresa varía entre los 3 y 7 años, y normalmente va ligado al momento en que la empresa ya es sostenible sin fondos adicionales. Es decir, que ha alcanzado el umbral de rentabilidad.

En el momento de salida del inversor social, la empresa puede tener dos tipos de rentabilidades. Por un lado, la sociedad y los aportantes de capital reciben la rentabilidad social, que es la que se busca maximizar. Adicionalmente, si hubiera un retorno financiero, este regresaría a los aportantes de capital en el fondo de capital riesgo filantrópico. En algunos casos, estos aportantes de fondos rein- 
vertirían sus retorno financiero de nuevo en inversión de impacto (Alemany y Scarlata, 2009). En la figura 2 se presenta el modelo de capital riesgo filantrópico.

Figura 2. Modelo de capital riesgo filantrópico (CRF)

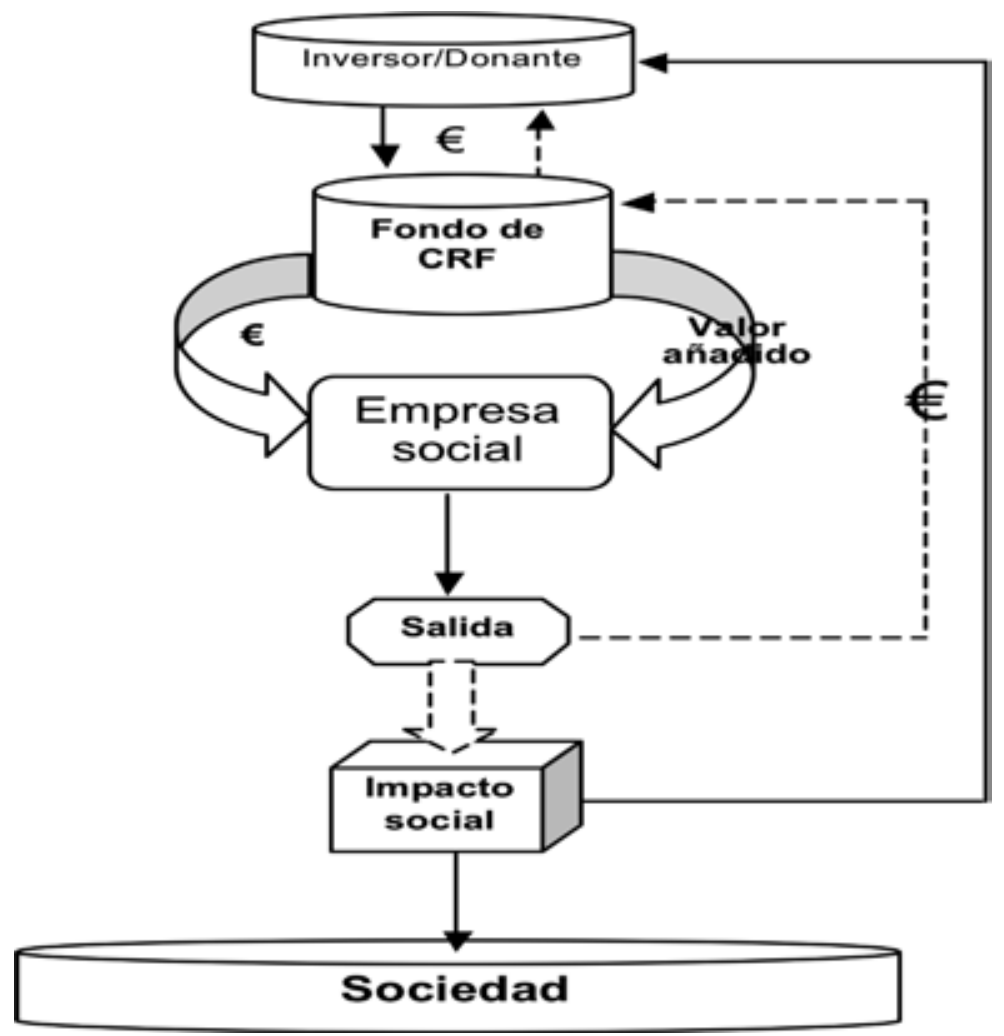

Fuente: Alemany y Scarlata (2009), pp. 147.

En la literatura existente sobre capital riesgo filantrópico se ha analizado y comparado la relación entre el modelo del capital riesgo tradicional y el del capital riesgo social. La pregunta que se estudia es si la inversión de impacto se enfrenta a los mismos problemas teóricos a los que se enfrenta el capital riesgo y, si es así, cuál es su comportamiento ante ellos.

Respecto a los temas que caracterizan el capital riesgo identificados en la literatura, la teoría de agencia, donde existe asimetría de información entre el emprendedor (agente) y el inversor (principal), es el más importante (Amit et al., 1993). El riesgo moral se da en aquellas situaciones en las que un individuo (el agente) sabe más que el otro sobre el efecto de sus propias acciones, pero el 
que soporta las consecuencias de estas acciones es el principal. Normalmente, el agente tiende a tomar más riesgo, ya que si sale mal será la otra parte la que asuma las consecuencias. Por ello, el inversor de capital riesgo desarrolla capacidades que le permiten minimizar el riesgo moral (Amit et al., 1998). En las operaciones de capital riesgo el inversor toma medidas, como la inversión por fases con consecución de hitos, la inversión mediante deuda convertible, en acciones preferentes o el establecimiento de un pacto de accionistas con cláusulas que le protegen, para minimizar el efecto del riesgo moral (Admati y Pfleidere, 1994; Sahlman, 1991; Hellmann, 1998).

John (2007) analiza el mercado Europeo de capital riesgo filantrópico y encuentra que la subvención o donación a fondo perdido es el instrumento más utilizado por los inversores. En el mismo estudio se presenta la inversión en fondos propios como un instrumento que cada vez se usa más. En datos recientes de la European Venture Philanthropy Association (EVPA) las subvenciones represen$\tan 73 \%$ del dinero aportado en el año 2010 por los encuestados, la deuda es $9 \%$ y la aportación vía fondos propios $11 \%$. Sin embargo, cuando se toman los datos de la encuesta más reciente de inversión de impacto de JP Morgan (2014), que analiza el mercado global, y sólo tiene en cuenta las "inversiones" (se excluyen las donaciones a fondo perdido), la deuda representa $62 \%$ del total de activos bajo gestión de este tipo de inversores (44\% deuda privada, $9 \%$ deuda pública y $9 \%$ deuda convertible/participativa), con fondos propios representando el $24 \%$. En este estudio se amplía considerablemente el ámbito de actuación, y el total de activos bajo gestión alcanza la cifra de 46 mil millones de dólares.

Además, en un estudio de Balbo et al. (2008) se concluye que los instrumentos utilizados por los inversores de impacto son similares a los que se utilizan en el mundo comercial, aunque se incorpora la subvención. Pero que en cualquier caso la rentabilidad de cualquier instrumento está siempre por debajo de la de mercado. Sin embargo, en el estudio reciente de JP Morgan (2014) 54\% de los encuestados esperan rentabilidades similares a las del mercado, $23 \%$ por debajo del mercado pero no mucho, y el restante $23 \%$ se conforman con la preservación del capital. Según estos resultados, se puede concluir que el instrumento utilizado por los inversores de capital riesgo filantrópico no es aquel que se adecua mejor a sus necesidades, si no a las circunstancias y necesidades de la empresa social, lo que de nuevo indica que el interés social va por delante del financiero.

Es por ello que la teoría del servidor ("Stewardship Theory", Donaldson y Davis, 1991; 1993) aparece en la literatura del capital riesgo filantrópico como contrapuesta a la teoría de agencia. La teoría del servidor, que tiene sus raíces en los campos de la sociología y psicología, caracteriza al ser humano por tener unas necesidades que van más allá de las económicas, como la autoestima, la necesidad de crecimiento y actualización o la necesidad de conseguir resultados. En este sentido, según la teoría del servidor, el agente (en este caso el emprendedor) 
dejado solo actuará de manera responsable en lo que respecta a los activos que él controla. Es decir, que no actuará en su propio interés y en contra del interés del principal (el inversor). Esta teoría se basa en el servicio, en contraste con el control de la teoría de agencia.

Si realmente la teoría del servidor se aplica en la relación entre el capital riesgo filantrópico y el emprendedor social, entonces el proceso de valoración de la compañía y el pacto de socios entre las partes debería ser diferente al del capital riesgo. Es decir, si la percepción del riesgo moral es baja, entonces en la estructuración de la operación de inversión no se negociará tanto la valoración y no habrá tantas clausulas donde se penalice al emprendedor, en caso de que las cosas no salgan según el plan, como las cláusulas de antidilución, las de preferencia en la liquidación, el vesting de las acciones o el derecho de arrastre. Según una investigación de Scarlata y Alemany (2011), cubriendo una muestra representativa del universo del capital riesgo filantrópico tanto en Estados Unidos como en Europa, la teoría del servidor describe mejor la relación entre el capital riesgo filantrópico y las empresas sociales. El riesgo moral percibido por el inversor es bajo, estableciendo sistemas de seguimiento menos intensivos que los del capital riesgo. Además, la palabra "confianza" aparece mencionada más frecuentemente que la palabra "control".

En este artículo se pretende estudiar la tensión entre los social y lo económico, y en particular si el vehículo de inversión de Momentum Project al analizar y decidir invertir en empresas sociales está más cerca de la teoría de la agencia, protección y control, o de la teoría del servidor, servicio al emprendedor social. Para ello se analizará el proceso de selección, la negociación, en concreto el tipo de cláusulas del pacto de socios, y por último el seguimiento de la empresa participada.

\section{METODOLOGÍA}

En este artículo se utiliza la metodología del análisis de caso longitudinal para estudiar el vehículo de inversión en empresas sociales de un banco español. El objetivo es entender la tensión creada entre el retorno económico y el social, y observar si es la teoría de la agencia o la del servidor la que mejor caracteriza a este inversor filantrópico. Además, el análisis del caso permite capturar la dinámica de la creación y la evolución de un fondo de inversión innovador dentro del mercado español, siendo el primero de este tipo, por lo que puede servir en el futuro como ejemplo a instituciones interesadas en poner en marcha fondos de inversión de impacto. Se trata de una investigación empírica.

El proceso de estudio es exploratorio, utilizando datos longitudinales y métodos cualitativos para aportar conocimiento en el reciente campo del capital riesgo filantrópico y la financiación de empresas sociales. Se han revisado informes 
internos del banco, desde el momento de la definición y creación de Momentum Project, hasta la fecha, incluyendo documentos sensibles como la evolución de las participadas del fondo o el pacto de accionistas que se firma entre el vehículo de inversión y la empresa social. Además, se han realizado entrevistas a altos directivos de los tres departamentos del banco implicados en Momentum: Responsabilidad Social Corporativa, de quien surge el proyecto y su liderazgo, Participaciones Societarias, responsable del vehículo de inversión y de su seguimiento, y Banca Privada, que tiene el rol de "vender" este producto financiero a sus clientes de grandes patrimonios.

Por último, los autores han participado en las cuatro ediciones de Momentum Project desde su creación hasta la fecha, año 2011 a 2014, habiendo tenido contacto tanto con los emprendedores, como con los estudiantes que apoyan los emprendimientos y con los mentores que añaden valor.

\section{CASO DE ESTUDIO, PARTE 1: MOMENTUM PROJECT}

\subsection{Inicios y objetivos del Programa Momentum}

En el año 2010 se crea el programa Momentum Project para ayudar al crecimiento de la empresa social en España. Programa que desde un inicio se convierte en un reto colaborativo para las dos instituciones impulsoras, un banco y una escuela de negocios. Y que se produce en un momento en que tanto las instituciones financieras como las escuelas de negocio estaban siendo muy cuestionadas por su responsabilidad en la crisis financiera. Al poco tiempo se suma al programa una de las grandes consultoras mundiales, lo que permitió consolidarlo aún más.

Así pues, Momentum Project lanza en el año 2011 su primera convocatoria con el objetivo de contribuir al desarrollo del emprendimiento social en España. La idea central era la implementación de un programa y un ecosistema de apoyo para que los emprendimientos sociales más prometedores pudieran consolidarse, crecer y escalar su impacto.

El programa desde sus inicios se marcó unos objetivos ambiciosos:

- Apoyar la consolidación y desarrollo de emprendimientos con soluciones efectivas y escalables para retos sociales y medioambientales existentes.

- Crear un ecosistema para los emprendimientos sociales y medioambientales.

- Fomentar la colaboración y el intercambio de conocimiento entre emprendedores, estudiantes y directivos para la creación conjunta de valor social y medioambiental.

- Generar conocimiento relativo al emprendimiento social y medioambiental. 
- Inspirar a la sociedad española a través de la difusión de casos de éxito y buenas prácticas de emprendimiento social y medioambiental.

- Propiciar la replicación del programa Momentum en otros países y regiones del mundo, una vez quede demostrada su efectividad.

- Fomentar y participar en la creación de un mercado regulado para financiar emprendimientos sociales y medioambientales.

\subsection{Las empresas sociales de Momentum Project y su proceso de selección}

Al ser un programa nuevo en España una de las primeras etapas fue definir muy bien el perfil de las empresas participantes. Momentum definió que una empresa social que participará en el programa había de ser una entidad privada que tenía el objeto de crear al mismo tiempo valor social y valor económico a través de iniciativas de mercado sostenibles. ${ }^{5}$

En la apertura de la primera convocatoria (2011) se concretaron en seis criterios específicos para la selección de empresas:

1. Impacto social relevante, sostenible económicamente, innovador y escalable

2. Dos años mínimo en operación (excepción en el caso de réplica de empresa internacional)

3. Dos empleados como mínimo

4. Actividad e impacto en España

5. Más de $100.000 €$ de ingresos anuales

6. Como máximo 49\% de ingresos anuales de donaciones y/o subvenciones.

Momentum Project decidió explícitamente apoyar a las empresas sociales que ya tenían un modelo de negocio probado en el mercado y que estaban en fase de crecimiento. Se pensó que las empresas en esta fase de su ciclo de vida eran las que podían crear más puestos de trabajo, tan necesarios en España. Naturalmente, está decisión hacía muy necesario desarrollar un buen proceso de selección. Este proceso se dividió en cuatro fases bien estructuradas.

En una primera fase se abría un período de inscripción on line donde los emprendedores sociales debían rellenar un formulario. El equipo de Momentum Project filtraba las candidaturas en base a los criterios de selección del programa. En estos primeros cuatro años se han inscrito en promedio más de 100 empresas sociales por edición, y han pasado el primer filtro la mitad. Las

\footnotetext{
${ }^{5}$ Esta definición es mucho más acotada que las utilizadas por otras organizaciones como por ejemplo Ashoka. Tanto el banco como la escuela de negocios participante tenían otras iniciativas para apoyar Fundaciones y ONG que trabajan a través de la filantropía. Momentum Project nació para consolidar organizaciones que trabajaban a través del mercado.
} 
que se han quedado en la primera mitad han sido porqué eran organizaciones filantrópicas. En una segunda fase las empresas sociales debían entregar más información sobre su modelo de negocio, la teoría de cambio que tenían y sobre diferentes aspectos financieros. Esta información era presentada vía internet por las empresas sociales con la ayuda de estudiantes. Un Comité de Preselección, compuesto por un equipo multidisciplinar con representantes de las instituciones organizadoras, seleccionaba a los 20 emprendimientos sociales que pasarían a la última fase. En esta etapa el equilibrio entre la creación de valor económico y valor social se miraba con detenimiento. En la última fase los emprendimientos tenían que presentar información financiera adicional, responder a preguntas de tipo fiscal y legal, y presentar un video resumen de un minuto. Finalmente, el Comité Científico, formado por expertos del sector social, empresarial, académico y financiero, seleccionaba los diez emprendimientos sociales que participarían en el Programa. Al principio el perfil del Comité era muy social y en la actualidad se ha equilibrado, entrando también perfiles empresariales.

\subsection{Contenido del programa Momentum}

Momentum Project nació con tres grupos de participantes que contribuían a hacer del mismo una experiencia única: emprendedores sociales, estudiantes y mentores. Y con tres elementos que ayudaban a configurar un programa muy completo: formación, acompañamiento y financiación.

\subsubsection{Emprendedores sociales, estudiantes y mentores}

Desde sus inicios el programa tenía como elemento central ayudar a los emprendedores sociales y sus empresas. El resto de componentes del programa eran facilitadores. Se trató de buscar la fórmula más idónea para conseguir este objetivo. Por eso el programa introdujo una primera innovación, respecto a otros proyectos similares, creando "comunidades de aprendizaje" dónde los emprendedores eran naturalmente el foco, pero a los que se sumaba la participación de estudiantes y de mentores. Los emprendedores sociales tenían que realizar el esfuerzo de pararse y reflexionar en profundidad sobre su actividad, pero se ponía a un equipo a trabajar con ellos. Momentum Project se basa pues en equipos de seis participantes, dos emprendedores sociales, dos estudiantes y dos mentores. Tener un equipo con el que los emprendedores pueden contrastar su experiencia permite elaborar planes de crecimiento muy detallados. Los estudiantes son los responsables de consolidar un documento estratégico sólido y los mentores de ayudar, gracias a su experiencia empresarial, a los estudiantes a ajustarse al máximo a la realidad del mercado. 


\subsubsection{Formación, acompañamiento y financiación}

La segunda innovación era que el programa se constituyó como "integral" 6 donde los emprendedores en principio podían obtener formación actualizada, un acompañamiento de mucha calidad, y si su plan de crecimiento convencía al banco, acceso a financiación en muy buenas condiciones. El que fuera un programa integral, no sólo lo hacía muy atractivo para los participantes, sino que permitía construir muchas sinergias entre las diferentes partes del mismo.

La formación sirve para que los emprendedores sociales tengan acceso a una serie de temas para comprender mejor la creciente complejidad de los mercados. Aspectos financieros y de acceso al capital, contenidos relacionados con la comercialización y las ventas, temas de innovación y creatividad son algunos de estos. Pero al mismo tiempo, el período de formación permite que los participantes puedan pararse y reflexionar sobre su emprendimiento. Y, en tercer lugar, la formación ayuda a crear un grupo compacto entre emprendedores, estudiantes y mentores, reforzando la idea de "comunidades de aprendizaje".

El acompañamiento, o "mentoring", se maduró como un elemento central. Se sabe que los emprendimientos sociales tienen muy pocas ocasiones de contrastar la creación de valor económico y social que sus empresas vienen realizando. Facilitándoles apoyo a través de un equipo se permite afrontar el crecimiento de sus empresas de una forma pausada y mucho más estructurada.

Por último, el banco consideró que si las empresas ofrecían planes de crecimiento interesantes era mejor que el dinero necesario viniera de un instrumento de financiación creado ad hoc para el programa. Por ello se decidió crear, como se detalla en el apartado 5, un instrumento de inversión de impacto.

\subsection{Las empresas participantes}

Desde su primera edición en 2011 son 40 emprendimientos sociales los que Momentum ha apoyado en España (ver figura 3). Actúan principalmente en el área de inclusión socio-laboral (65\% de ellos), lo que sigue la lógica del programa por apoyar a empresas que dan empleo a personas en riesgo de exclusión social (con discapacidad, mujeres inmigrantes con escasos recursos, personas que salieron de la cárcel, etc.) en un periodo en que las tasas de desempleo para la población española, y estos colectivos en particular, han sido extremadamente altos.

\footnotetext{
${ }^{6}$ El elemento de financiación por parte del banco no estaba previsto en el lanzamiento del Programa, pero durante la fase de acompañamiento se vio que tenía sentido crear un vehículo de financiación para el mismo.

${ }^{7}$ También ha apoyado a 20 empresas sociales en México, y a 6 en Perú. En este artículo se analizarán sólo las empresas y el instrumento de inversión del programa en España.
} 
Figura 3. Áreas de impacto social empresas

\section{Areas de impacto social}

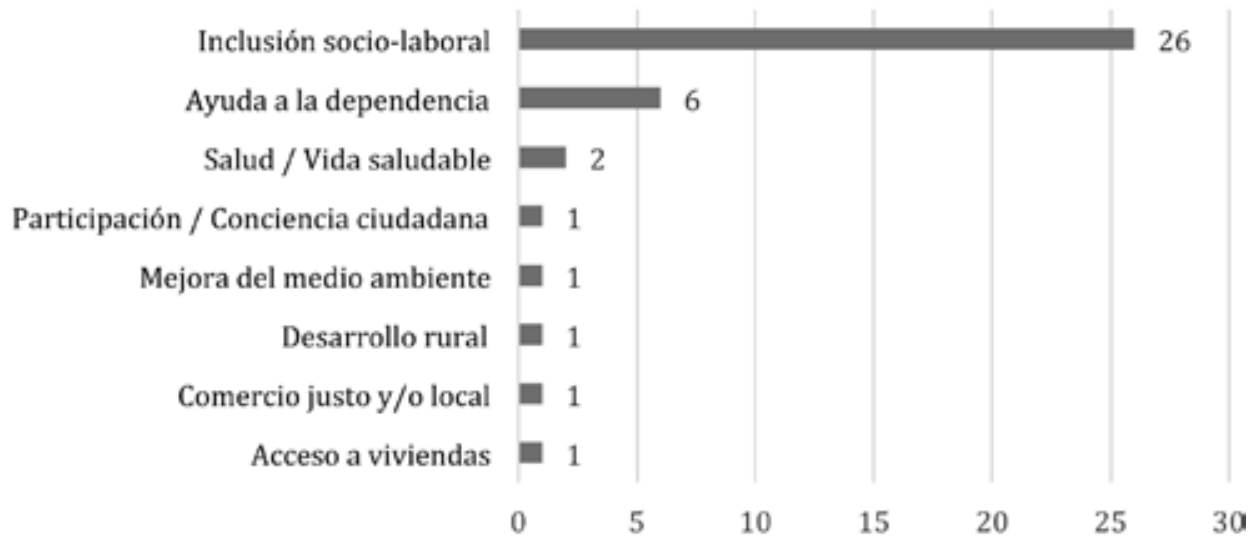

Fuente: Momentum, 2014, pp. 5.

En cuanto a la creación de valor económico, los emprendimientos de Momentum, actúan en distintos sectores de la economía, siendo el sector de servicios el que más destaca seguido del sector alimentación y bebidas (ver figura 4).

\section{Figura 4. Áreas de impacto social empresas Momentum, número de empresas.}

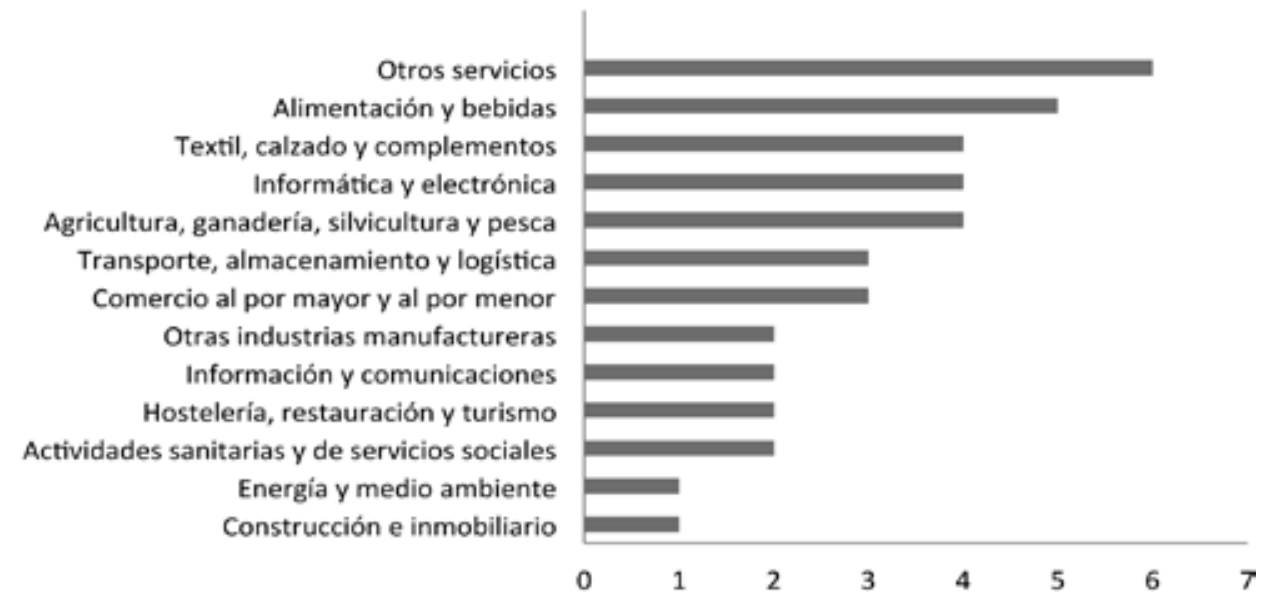

Fuente: Momentum, 2014, pp. 6. 
En el año 2014, las empresas de Momentum Project han facturado más de 35 millones de euros, con beneficios medios de $31.587 € .{ }^{8}$ El número de beneficiarios totales es de 1.146 .059 personas. Por último, en 2014 empleaban a 926 personas. De éstas 555 eran personas en inserción socio laboral, es decir un 60\% del total de empleados. Por otro lado 179 eran jóvenes de menos de 30 años, representando un $19 \%$ del total de empleados.

\section{CASO DE ESTUDIO, PARTE 2: MOMENTUM SOCIAL INVESTMENT, SL}

\subsection{Creación y evolución del vehículo de inversión}

Una vez que Momentum Project está en marcha en la primavera del año 2011, el equipo de liderazgo decide contactar con las áreas de Banca Privada y Participaciones Societarias del banco. Banca Privada para sondear el potencial interés de sus clientes en participar en el proyecto, y Participaciones Societarias para ver cómo se podría diseñar un vehículo para invertir en las empresas sociales apoyadas por el programa Momentum. Esta petición es realmente novedosa, sin embargo, para la dirección de ambas áreas, una vez entendido el alcance y los objetivos de Momentum, tiene sentido y encaja con los intereses del banco y de sus clientes, que tienen interés en temas filantrópicos y además les permite diversificar su inversión en otro tipo de empresas.

Inicialmente se evalúan 4 modelos para el vehículo de inversión de impacto:

1. Vehículo regulado: fondo de capital riesgo (FCR) o sociedad de capital riesgo (SCR) regulado por la CNMV. Sin embargo, por el tamaño mínimo del fondo, por la limitación del FCR o SCR a la inversión a través de préstamos, y por las obligaciones que representa estar regulados por la CNMV, se decide que la ventaja fiscal no compensa el resto de desventajas.

2. Vehículo filantrópico, tipo fundación o asociación: encaja bien en la filosofía de inversión en empresas sociales, sin embargo presenta desafíos. Por un lado, en una fundación hay que tener un patronato y tal vez no es lo más eficiente para la toma de decisiones de inversión. Además, la idea del vehículo de inversión de impacto es que pueda tener beneficios, de manera que pueda seguir apoyando a más empresas sociales en el futuro. Los vehículos filantrópicos tienen limitaciones en cuanto al reparto de beneficios.

${ }^{8}$ Los datos de 2014 son estimados al cierre del año para un total de 33 emprendimiento (82,5\%) del conjunto del portfolio desde 2011. 
3. Sociedad Anónima (SA): encaja, pero finalmente se determina que la SL es más eficiente.

4. Sociedad Limitada (SL): en un principio no está claro que esta forma mercantil permita hacer inversión vía préstamos retribuidos, sin embargo, una vez evaluado se ve que es factible. La SL tiene otras dos ventajas respecto a la SA, por un lado como vehículo es más sencillo de manejar y por otro el Consejo de Administración es más pequeño, lo que permite la toma de decisiones rápida.

Finalmente se opta por la opción SL. Una vez decidida la forma societaria del vehículo se abren otros dos temas, uno de gobierno corporativo de la SL y otro respecto a quién dentro del banco debe de ser el propietario de la participación en el vehículo de Momentum Social Investment SL (MSI, SL).

Respecto al tema del gobierno corporativo del vehículo surgen algunas preguntas: ¿quién tiene que estar en el Consejo de Administración? ¿Tiene sentido que los clientes estén en los órganos de gobierno? Desde el principio, el objetivo del vehículo es que sea ágil y eficiente, que la toma de decisiones sea rápida.

El consejo tendrá 2 comités asesores:

- Comité de inversión y seguimiento de participadas: selección de inversiones nuevas y seguimiento de empresas ya invertidas. Compuesto por miembros del banco (RSC, Banca Privada y Participaciones Societarias), la escuela de negocios y la consultora.

- Comité de ejecuciones: decisiones de ejecuciones de préstamos en casos de fraude o incumplimientos graves por parte de las empresas participadas.

Una vez resuelta la estructura y organización societaria es necesario identificar la tipología de clientes interesados en coinvertir con el banco en el vehículo de inversión social.

\subsection{Estructura, objetivos y proceso de análisis del vehículo}

El plan inicial del vehículo de inversión Momentum Social Invesment SL es de una SL por año del Programa Momentum. Sin embargo, a partir de 2014, se decide constituir una sociedad holding que previsiblemente se fusionará con las anteriores durante 2015, con el objetivo de tener una sola entidad a gestionar. Los accionistas de las SL son por una parte el banco, a través de Participaciones Societarias, y por otra parte sus clientes. La SL invierte en aquellas empresas "graduadas" de Momentum que necesitan fondos y que pasan el análisis de inversión que se detalla más adelante. La inversión puede ser en fondos propios o en deuda. 
Los objetivos que se fijan son dobles, algo habitual en el sector de impacto. Por un lado se busca el impacto social, y por otro el retorno financiero. Respecto al impacto social se pretende maximizar su alcance y buscar estructuras que tengan un efecto multiplicador de este impacto social. Este primer objetivo es una condición necesaria, aunque no suficiente. En referencia al retorno financiero el objetivo principal es la recuperación del capital, que permita asegurar la viabilidad del vehículo en el largo plazo. De esta manera se asegura la sostenibilidad de Momentum Social Investment y se puede continuar generando un mayor impacto social. Además, se busca que las empresas sean sostenibles y escalables, lo que de nuevo garantiza el éxito a largo plazo del proyecto y maximiza el impacto.

Las empresas sociales que quieren acceder a la financiación de MSI SL pasan tres etapas de selección:

1. Selección para el programa Momentum: el Comité Científico (ver apartado 4.2), formado por representantes del mundo social, académico e inversor, se reúne anualmente y elige a los diez emprendimientos sociales de ese año. El Comité Científico utiliza como parámetros en su selección el impacto social actual y potencial, el modelo de negocio, teniendo en cuenta su sostenibilidad y escalabilidad, y finalmente las expectativas de crecimiento.

2. Información de las compañías participantes: se elabora un cuaderno de información, donde de manera resumida se incorpora toda la información clave de la compañía y sus plan de negocio de crecimiento, donde se identifican las necesidades, tanto financieras como de otro tipo de recursos, para alcanzarlo. Este cuaderno de información es preparado por el banco, la consultora y la escuela de negocios.

3. Una vez el Cuaderno de Información está listo, las empresas son invitadas a presentar en el Foro de Inversión Social Momentum, que tiene lugar en otoño, después que las empresas seleccionadas han pasado todo el proceso de formación y mentoring y los alumnos han elaborado su plan estratégico de crecimiento durante el verano.

Una vez finalizado el Foro de Inversión Social de Momentum, el equipo de Participaciones Societarias comienza el análisis de los proyectos, cuyos resultados serán presentados al Comité de Inversiones de MSI SL, para que decida en cuales invertir:

1. Se analiza el plan de inversiones propuesto y se complementa la información con la due diligence preparada por el equipo de la consultora.

2. Análisis financiero y de riesgo de la información entregada por la compañía: se realiza un estudio de la proyección financiera, con el objetivo de 
entender la capacidad de repago, en caso de inversión vía préstamo, los riesgos del negocio, y sacar conclusiones, desde el ángulo financiero, del plan presentado.

Propuesta de Inversión, similar a lo que en el sector de capital riesgo se denomina el "investment memorandum", es un documento preparado por Participaciones Societarias de banco que incluye la siguiente información: descripción de la compañía y modelo de negocio; equipo y organización; mercado y competencia; plan de empresa; destino de la inversión solicitada; datos financieros y capacidad de repago; impacto financiero y social.

\subsection{Instrumento de Inversión}

Como se ha mencionado con anterioridad, el vehículo de inversión social se estructura para poder invertir en las empresas vía deuda o vía fondos propios.

\subsubsection{Deuda}

En la mayoría de los casos la inversión se materializa vía préstamo. Hay tres razones para ello. Primera, la razón social de la empresa dificulta la entrada en el capital social. Es el caso de las fundaciones, asociaciones o cooperativas. Segunda, la dificultad para desinvertir. Son casos donde es poco probable que la compañía se pueda vender en el futuro, lo que limita la salida del accionista y por lo tanto la rentabilidad financiera. Por último, es el propio emprendedor social el que no quiere tener otros socios en el accionariado. Puede ser por temor a perder el control o por no tener costumbre, por lo que son los emprendedores los que solicitan la inversión vía préstamo.

Se trata de un préstamo con carencia de cuatro años en el repago del principal, que se amortiza en los siguientes cuatro años (del año 5 al 8, ambos incluidos), a partes iguales, $25 \%$ anual. El tipo de interés es creciente, comenzando con un $2 \%$ durante los dos primeros años, que son clave en la ejecución del plan de negocio, aumentando a $4 \%$ durante los años 3 y 4 , y con un incremento de un $1 \%$ anual hasta llegar al $8 \%$ en el último año de cancelación total del principal. Hay previstas primas de rentabilidad en el caso de venta de la empresa y de devolución anticipada, y si la empresa tiene beneficios superiores a los previstos en el plan.

\subsubsection{Fondos Propios}

En algunos casos la inversión se estructura en forma de inversión en el capital de la empresa, a través de la compra de acciones de nueva emisión. Para ello tienen que darse las tres condiciones necesarias. Primera, que la forma jurídica de 
la empresa lo permita, es decir, que sea una SA o SL. Segunda, que se consideré posible la venta futura de la participación a un tercero o al propio emprendedor (el emprendedor lo acepta y el plan de crecimiento es atractivo). Y por último, que el emprendedor quiera la entrada de nuevos socios en el capital.

Si la inversión se estructura vía fondos propios MSI SL tiene los mismos derechos que cualquier accionista durante los primeros cinco años. A partir del año seis, MSI SL tiene derecho a la venta, o amortización vía reducción de capital, de un tercio de su participación durante cada uno de los tres años siguientes, es decir, que en el año 8 tendría derecho a desinvertir un 100\% de su inversión.

Respecto a la valoración de la participada, se estipula en el pacto de accionistas que a la salida el inversor tenga una rentabilidad mínima garantizada, por lo que se traslada la negociación sobre el valor de la empresa al momento de liquidez futuro.

\subsection{El Pacto de Socios}

El acuerdo de inversión y el pacto de socios del vehículo MSI SL, contiene las cláusulas habituales en lo que respecta a representación en el Consejo de Administración, mayorías reforzadas para temas clave (como cambios en los estatutos, la estructura de capital, fusiones y adquisiciones, venta de acciones, cambios en los consejeros o en los auditores), obligación de auditar las cuentas, derecho de información y pacto de salida a partir del año 6 .

Sin embargo, no se incorporan clausulas estándar del capital riesgo como las de arrastre, las de preferencia en el caso de venta, las de antidilución, o las de "vesting" de las acciones de los emprendedores.

\subsection{Seguimiento y control de la cartera}

Para el seguimiento de las inversiones se establece un sistema con tres niveles: 1.- Responsable del seguimiento; 2.- Comité de seguimiento y 3.- Consejo de Administración.

La persona responsable del seguimiento recibe los informes trimestrales de la empresa. Estos informes son analizados con expertos financieros y sociales, ya que la evolución del impacto social es clave. Desde el punto de vista financiero el objeto de análisis es asegurar que no hay ninguna alarma que pueda indicar que se dará una situación complicada en el corto o medio plazo. El responsable informa al Comité de Seguimiento.

El Comité de Seguimiento es responsable del seguimiento y asesoramiento al Consejo en la toma de decisiones. Se reúne semestralmente. Está compuesto por miembros del banco (Participaciones Societarias, Banca Privada, y Responsabilidad Social Corporativa), uno de la consultora y uno de la escuela de negocios. 
Por último, el Consejo de Administración del vehículo está compuesto por tres miembros del banco, que son responsables del gobierno y la toma de decisiones del vehículo (pendiente de modificación con la entrada de inversores).

\section{RESULTADOS DEL ANÁLISIS Y LECCIONES APRENDIDAS}

El objetivo principal de este artículo es analizar el equilibrio entre el retorno social y el financiero, y que papel juegan estos retornos en el programa Momentum y su vehículo de inversión MSI SL. Para ello se realizan dos análisis. En el primer análisis, se examinan las empresas que han obtenido financiación, clasificándolas según sus características y colocándolas en el continuum nonprofit-forprofit presentado en la revisión bibliográfica. De esta manera podemos ver donde se sitúa Momentum. En el segundo análisis, se centra en el instrumento de inversión, fondos propios vs. deuda, y sus características, con el que se han financiado las empresas sociales.

\subsection{Empresas de Momentum que han obtenido financiación}

Después del análisis de las 40 empresas y organizaciones que han pasado por Momentum en los primeros 4 años del programa (2011 a 2014 incluido), se pueden establecer 4 grupos entre las empresas participantes (ver Figura 5).

Grupo 1: 8 asociaciones y fundaciones que tienen una actitud emprendedora, algunas inclusive han creado una empresa social que gestiona el emprendimiento social. Como hemos analizado en la revisión bibliográfica muchas organizaciones no lucrativas con la crisis han buscado nuevas formas de obtener fondos. Sin embargo, en el programa Momentum estas organizaciones no han recibido financiación. La mitad de ellas por qué al final de todo el programa dijeron que no estaban interesadas en la misma y no solicitaron fondos. Y la otra mitad porqué el banco no vio el emprendimiento interesante.

Grupo 2: 9 asociaciones y fundaciones que han creado una empresa social, y han dado autonomía para que esta empresa gestione el emprendimiento. En este caso, el banco ha visto claramente la oportunidad de invertir en estas empresas. Es un grupo que tiene su nacimiento en sector no lucrativo pero que ha evolucionado con la creación de una "spin-off".

Grupo 3: 10 empresas sociales que se han creado bajo el paraguas jurídico de una cooperativa, sociedad limitada o sociedad anónima. En estas el banco también ha decidido invertir. Sus fundadores han expresado claramente una vocación social, y ven el emprendimiento como un instrumento para generar mayor impacto.

Grupo 4: 9 sociedades anónimas o limitadas cuya preocupación principal es la financiación para escalar su negocio, no tanto la motivación social. Son empresas 
que tienen una preocupación social, pero es menos importante que en el grupo 3. El banco sólo ha financiado una empresa de este grupo.

\section{Figura 5. Grupos de empresas participantes según continuum nonprofit - forprofit El continuum nonprofit - forprofit}

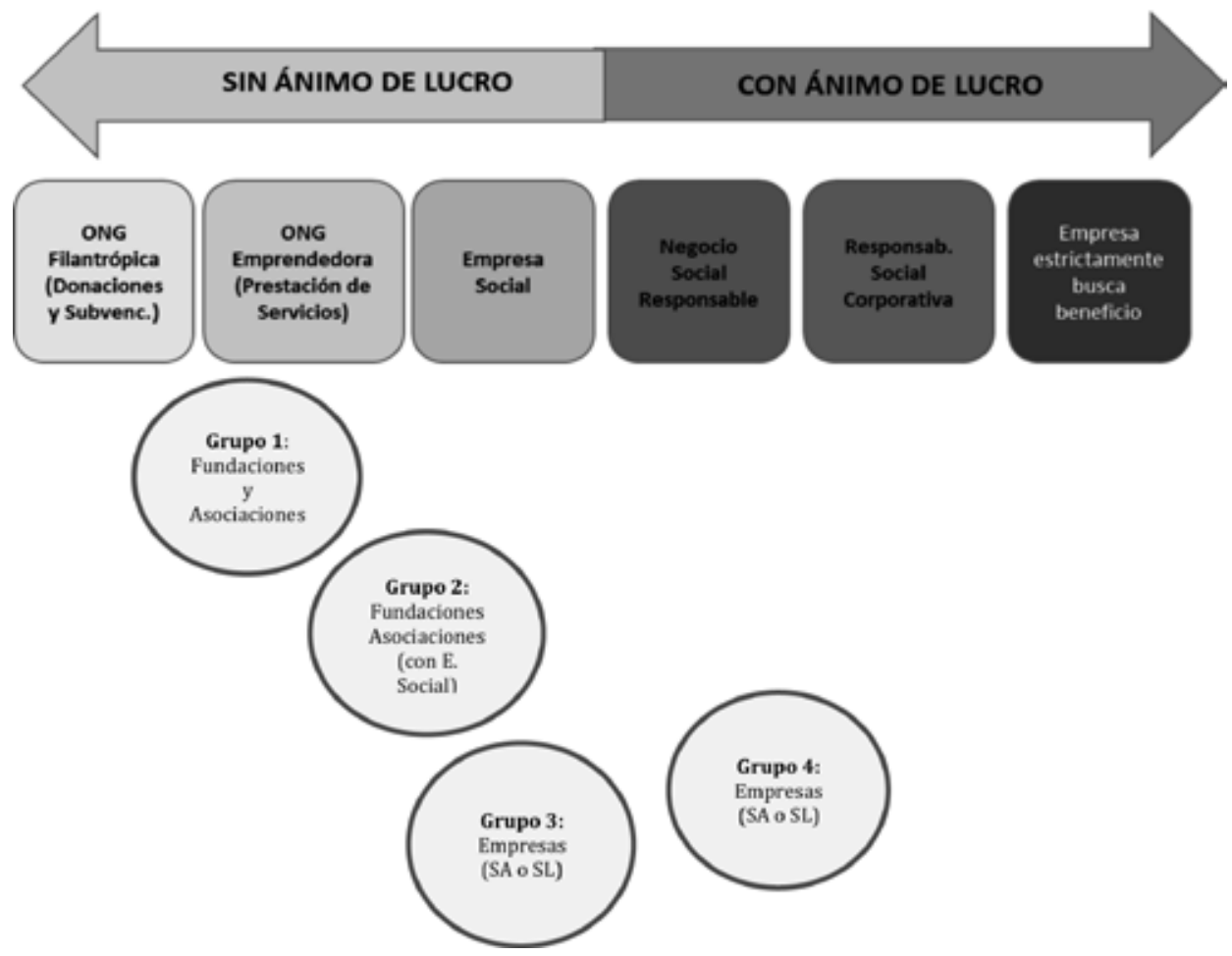

Fuente: Elaboración propia.

Como se puede observar de los resultados del análisis, Momentum se centra en la financiación de empresas en la frontera de lo social y el ánimo de lucro. Es decir, no financia empresas puramente sociales sin ánimo de lucro, si no empresas sociales que están en cercanas a la empresa tradicional de mercado. Y no pasa prácticamente la frontera, salvo en una ocasión, en la que se financia una empresa con ánimo de lucro con una componente social en el ámbito de la responsabilidad social corporativa. Además, esta empresa es de la primera edición, donde todavía se estaba definiendo bien el enfoque del vehículo de inversión de impacto, y se puede afirmar que en la actualidad el propósito es no pasar esa frontera del continuum. 


\subsection{Instrumento de inversión y condiciones}

Analizando el instrumento de inversión se observa que en todos los casos menos en una empresa, la financiación ha sido otorgada a través de un préstamo. En la sección 5.3.1 se detallan los distintos motivos para instrumentar la inversión vía deuda. En las entrevistas realizadas los 2 motivos que predominan es que o bien la forma societaria de la empresa no permite entrada vía capital (como en el caso de las cooperativas o fundaciones) o el emprendedor no quiere tener otros socios y por lo tanto prefiere préstamo a inversión en fondos propios.

Las condiciones del préstamo, tanto por el periodo de carencia de cuatro años, como por el interés más bajo en los primeros años, cuando es más complicado para la empresa hacer frente a los pagos, y sin pedir garantía, son favorables al emprendedor.

Por lo tanto, tanto la motivación para el préstamo, que depende del emprendedor social y no del banco, como las condiciones de éste, parecen apoyar la teoría del servicio frente a la teoría de agencia. Sin embargo, es interesante resaltar que el préstamo se beneficia en el caso de una venta de la empresa o de beneficios superiores a los previstos en el plan, es decir, que se trata de un préstamo participativo, lo que le hace cercano a los fondos propios (denominado "quasi-equity"). Es esta parte de participación en los beneficios, que no son esperados, pero que podrían darse, que el vehículo de inversión de impacto MSI SL se comporta como un capital riesgo filantrópico, y de nuevo vemos esa búsqueda del equilibrio entre lo social y lo financiero. Sin embargo, para que el vehículo Momentum Social Investment consiga su doble objetivo de retorno financiero y social se tienen que hacer más inversiones en fondos propios. Para ello es necesario que se den más empresas que cumplan las condiciones necesarias para este tipo de inversión: forma jurídica adecuada, plan de crecimiento atractivo, buena relación con el equipo, que esté abierto a este tipo de inversión y finalmente que pueda haber opciones para la salida.

El análisis del caso Momentum muestra que la principal barrera son los emprendedores que no ven la deuda de la misma manera que los fondos propios. El concurso de acreedores da respeto, pero el retraso o incumplimiento en el pago de la deuda es diferente. Además, al tratarse de deuda, en la mayoría de los casos, no se tiene la sensación de que se tiene un socio que quiere ayudar y añadir valor "no es la misma presión el contar con un nuevo socio en el Consejo y que pida cosas a la presión de pedir información, previsiones o presupuestos si han recibido la financiación vía deuda", afirma un empleado del banco. Este resultado contrasta con la intención del inversor de "prestar servicio", ayudando para que la empresa esté gestionada de una manera más cercana al de la empresa tradicional, con la visión del emprendedor que lo ve como una carga adicional de trabajo.

Una de las principales vías para añadir valor es a través de la asesoría legal y durante el proceso de due diligence, ya que se pueden identificar puntos débiles a 
mejorar. Así mismo, la implementación de un buen sistema de control financiero interno de las participadas es una herramienta que sirve para la toma de decisiones en la gestión. Sin embargo, la mayoría de los emprendedores sociales "a la larga le verán el valor de tener información trimestral, pero de momento no lo valoran" apuntan desde el departamento de Participaciones Societarias. Además, no se trata solo del seguimiento financiero, se trata de una inversión de impacto, por lo que el valor añadido es parte fundamental del paquete ofertado. Es como el capital riesgo, necesita un equipo dedicado, que pueda ayudar a la participada en las decisiones estratégicas y en la provisión de contactos. Si no se añade valor entonces no está claro que sea una inversión de impacto.

\section{CONCLUSIONES}

Tanto la estructura como la gestión del vehículo han evolucionado con Momentum Project. Desde la primera edición en el año 2011, todo el proceso se ha revisado anualmente para identificar factores a mejorar, con el objetivo de tener un mayor impacto, especialmente bajo el ángulo de ayudar a las empresas sociales que participan en el programa. Los comentarios de las empresas y su evolución han servido al equipo de liderazgo de Momentum Project para poder aprender y mejorar.

Los primeros años de Momentum Project han estado marcados constantemente por la tensión entre la creación de valor económico y valor social. Tensiones que se han dado a diferentes niveles y en los diferentes stakeholders.

Primero dentro de las propias empresas sociales. A los emprendedores que han nacido en organizaciones con clara vocación social, al tener que interactuar con el mercado les han provocado constantes problemas internos. Y, viceversa, las empresas que han nacido con una clara vocación de enfrentarse al mercado, en un momento de crisis económica, la búsqueda del equilibrio no les ha sido nada fácil. El "capital paciente" de MSI SL se ha mostrado muy útil. Estas tensiones internas serán interesantes de analizar en mayor profundidad para algunas de las empresas participantes.

En segundo lugar dentro del banco. En este caso, el departamento de responsabilidad social corporativa ha tenido que mediar con el departamento de Empresas Participadas, que es quién gestiona el Fondo. De nuevo los intereses pueden parecer opuestos, aunque el éxito reside en la búsqueda del equilibrio.

También en la empresa de consultoría, que tiene una Fundación que entiende mejor la parte social y que está dentro de su misión, y unos socios empresariales que están por la creación del valor económico.

Y, finalmente, en la universidad, donde el programa de formación y de mentoring está a caballo entre dos Institutos: el de Innovación Social, y el de Iniciativa Emprendedora. 
Desde que arrancó con fuerza la inversión de impacto muchos inversores, académicos e intermediarios han estado preocupados por el tamaño de las empresas sociales. Es verdad que existen muchas empresas sociales, pero pocas alcanzan un tamaño significativo, lo que hace difícil dirigir fondos hacia ellas. El cómo generar escala en las empresas sociales se ha convertido en un tema central en la literatura. ${ }^{9}$ Seguramente, uno de los aspectos más interesantes de tener un programa integral es que permite conocer muy bien a cada una de las empresas sociales participantes. Aunque es pronto para realizar este tipo de análisis, se pueden realizar algunas hipótesis sobre las dificultades que tienen para escalar las empresas Momentum.

Dentro de las lecciones aprendidas hasta la fecha también se pueden mencionar los dos grandes desafíos que presentan los equipos y la búsqueda de la sostenibilidad financiera. En lo que se refiere a los recursos humanos, algunas empresas sociales tienen problemas para localizar personas preparadas para sus órganos de gobierno, otras para encontrar directivos para afrontar con solidez la gestión de la entidad, y también las hay que no tienen la capacidad de preparar el personal operativo necesario con la suficiente rapidez. Respecto a los equipos emprendedores que mejor funcionan, son aquellos que se componen de un tándem. Uno de los emprendedores tiene la visión social y busca como incrementar su impacto. El otro está más centrado en la gestión de la empresa, en el día a día de los ingresos y los gastos. Esto es similar a lo que se ha observado en industrias donde la creatividad es clave, como en la arquitectura, el cine o la alta cocina (Svejenova et al., 2006; Svejenova y Vives, 2009).

El segundo elemento que está entorpeciendo el desarrollo de las empresas es su capacidad de generar valor económico, lo que no permite muchas veces generar más valor social. Esto puede tener varias causas, pero una de las principales que citan las empresas participantes es la dificultad de incrementar las ventas de sus productos/servicios en el contexto de crisis económica del país.

Seguramente los dos elementos mencionados anteriormente están íntimamente relacionados. Si una empresa social no genera suficientes recursos económicos, va a tener dificultades para atraer talento. Es por este motivo que en la elección de las empresas de Momentum cada vez más se está prestando más atención al liderazgo de las mismas y a sus equipos.

Otro factor citado por varias empresas, es la no priorización del crecimiento de la empresa por parte de su órgano de gobierno. El órgano de gobierno tiene como responsabilidad encontrar la armonía entre la creación de valor económico y valor social. Este equilibrio no es nada fácil de conseguir en empresas sociales de tamaño pequeño. Esto muchas veces está ligado al miedo a perder la misión social

\footnotetext{
${ }^{9}$ Ver por ejemplo la excelente revisión de la literatura realizada por C. Clark, C. Massarsky, T. Raben y E. Worsham (2012), Scaling Social Impact: A Literature Toolkit for Funders.
} 
de la empresa. Lo que en la literatura anglosajona se conoce como "misión-creep" (Moore, 2000). Algunos de los factores mencionados son consistentes con los hallazgos de Bloom y Chatterji (2009), y abren una interesante vía de investigación de cara al futuro.

Por último, las principales lecciones aprendidas del equipo que gestiona el vehículo de inversión, comparando estas inversiones de venture philanthropy con el capital riesgo tradicional, son:

- Es más fácil cerrar una operación. En el capital riesgo hay que evaluar muchos factores, hay que tener en cuenta todos los factores de riesgo. Hay que valorar la empresa y negociar las condiciones. En el caso de la inversión de impacto, el análisis previo al cierre de la inversión se centra más en ver que la empresa es sostenible.

- En la actualidad en este tipo de inversiones no hay competencia. Esto es muy diferente en el sector del capital riesgo, donde en algunos casos hay "peleas" por invertir.

- En la inversión de impacto se toman muchas menos precauciones, en el sector social no se piensa que te pueden engañar, se genera más confianza, no se piensa en los "limones".

- En el capital riesgo tradicional la negociación con el equipo emprendedor es uno contra otro. En la inversión de impacto se pone por delante de todo el retorno social y se piensa en como maximizarlo haciendo crecer el negocio.

- En la emprendeduría tradicional prima el equipo, en el mundo social se da más el emprendedor solitario.

- Por último, se ha decidido abrir el vehículo a proyectos más allá de Momentum Project, para incrementar el impacto social al máximo, y centrar mayores esfuerzos en potenciar la colaboración y la inversión en proyectos ya invertidos con el objetivo de crear "Campeones Sociales" en la industria del Sector Social en España.

\section{BIBLIOGRAFIA}

ADMATI, A. R. y PFLEIDERE, P. (1994), "Robust financial contracting and the role of venture capitalists", Journal of Finance, vol. 49, núm. 2, pp. 371-402.

ALEMANY, L. y SCARLATA, M. R. (2009), "Philanthropic venture capital: a new model of financing for social entrepreneurs", en D. Cumming (ed.), Companion to Venture Capital. Wiley Press, Nueva York, pp. 131-149.

AMIT, R., GLOSTEN, L. y MULLER, E. (1993), "Challenges to theory development in entrepreneurship research", Journal of Management Studies, vol. 30, núm. 5, pp. 815-834. 
AMIT, R., BRANDER, J. y ZOTT, C. (1998), "Why do venture capital firms exist? Theory and Canadian evidence", Journal of Business Venturing, vol. 13, núm. 6, pp. 441-446.

BALBO, L., MORTELL, D. y OOSTLANDER, P. (2008), "Establishing a venture philanthropy fund in Europe", EVPA Publications, Brussels.

BATTILANA, J. y otros (2012), "In search of the hybrid ideal", Stanford Social Innovation Review, vol. 10, núm. 3, pp. 51-56.

BLOOM, P. N. y CHATTERJI, A. K. (2009), "Scaling social entrepreneurial impact", California Management Review, vol. 51, núm. 3, pp. 114-133.

CLARK, C., y otros (2012), "Scaling social impact: a literature toolkit for funders". Growth Philanthropy Network and Duke University.

DEES, G. (1998), "The Meaning of Social Entrepreneurship" accessible en: http://www.caseatduke.org/documents/dees_sedef.pdf.

DEES, G y ANDERSON, B. (2006), "Framing a Theory of Social Entrepreneurship: Building on Two Schools of Practice and Thought" en Research on Social Entrepreneurship: Understanding and Contributing to an Emerging Field. ARNOVA Occasional Paper Series 1, núm. 3.

DONALDSON, L. y DAVIS, J. H. (1991), "Stewardship theory or agency theory: CEO governance and shareholder returns", Australian Journal of Management, vol. 16, núm. 1, pp. 49-64.

DONALDSON, L. y DAVIS, J. H. (1993), "The need for theoretical coherence and intellectual rigor in corporate governance research: reply to critics of Donaldson and Davis", Australian Journal of Management, núm. 18, pp. 213-225.

EMERSON, J. y TWERSKY, F. (1996), "New Social Entrepreneurs: The Success, Challenge, and Lessons of Non-profit Enterprise Creation", Roberts Foundation, San Francisco.

GOMPERS, P. y LERNER, J. (2001), "The venture capital revolution”, Journal of Economic Perspectives, vol. 15, núm. 2, pp. 145-168.

HELLMANN, T. (1998), "The allocation of control rights in venture capital contracts", RAND Journal of Enconomics, vol. 29, núm. 1, pp. 57-76.

JOHN, R. (2007), "Beyond the cheek: How venture philanthropists add value", Said Business School Publications, Oxford.

J. P. MORGAN (2014), Spotlight on the market: the impact investor survey, New York.

LEADBEATER, C. (1997), The rise of the social entrepreneur, Demos, New York.

LETTS, C. y otros (1997), "Virtuous Capital: What foundations can learn from venture capitalists", Harvard Business Review, vol. 75, núm. 2, pp. 36-44.

MAIR, J. y MARTI, I. (2006), "Social entrepreneurship research: A source of explanation, prediction, and delight", Journal of World Business, vol. 41, núm. 4, pp. 36-44. 
MARTIN, L. y OSBERG, S. (2007), "Social entrepreneurship: the case for definition", Stanford Social Innovation Review, vol. 5, núm. 2, pp. 28-39.

MARETICH, M. y BOLTON, M. (2010), "Social Enterprise: From Definitions to Developments in Practice", EVPA, Bruselas.

MÁRQUEZ, P. y otros (2010), Socially inclusive business: Engaging the poor through market initiatives in Iberoamerica. Harvard Business Publishing, Cambridge.

MOORE, M. (2000), "Managing for Value: Organizational Strategy in forProfit, Nonprofit, and Governmental Organizations", Nonprofit and Voluntary Sector Quarterly, vol. 29, núm. 1, pp. 183-204.

MURPHY, P., and COOMBES, S. (2009), "A model of social entrepreneurial discovery”, Journal of Business Ethics, vol. 87, núm. 3, pp. 325.

NORRIS, W. C. (1983), New frontiers for business leadership, Dorn Books Minneapolis, MN.

OSBORNE, D. y GAEBLER, T. (1992), Reinventing Government. How the entrepreneurial spirit is transforming the Public Sector, Addison-Wesley Publ. Co., Boston.

PEREDO, A. y McLEAN, M. (2006), "Social entrepreneurship: A critical review of the concept", Journal of World Business, vol. 41, núm. 1, pp. 56-65.

PONTIKES, E. (2012), "Two sides of the same coin. How ambiguous classification affects multiple audiences' evaluation”, Administrative Science Quarterly, vol. 57, núm. 1, pp. 81-118.

REED, L. y otros (2014), Resilencia. El Informe del Estado de la Campaña de la Cumbre de Microcrédito 2014, Microcredit Summit Campaign, Washington DC.

SAHLMAN, W. A. (1991), "Aspects of financial contracting in venture capital", Journal of Applied Corporate Finance, núm.1, pp. 23-36.

SCARLATA, M. R. y ALEMANY, L. (2008), "Financing Social Entrepreneurs: Philanthropic Venture Capital vs. Foundations", BERC conference, Chapel Hill, NC.

SCARLATA, M. R. y ALEMANY, L. (2010), "Deal structuring in philanthropic venture capital investments: financing instrument, valuation and covenants", Journal of Business Ethics, núm. 95, pp. 121-145.

SKLOOT, E. (1983), "Should not-for-profits go into business?", Harvard Business Review, vol. 61, núm. 1, pp.20-27.

SVEJENOVA, S., PLANELLAS, M. y TERJESEN S. (2006), "Dynamics of entrepreneurial teams in ventures in creative industries", BCER Conference, Babson College, 2006.

SVEJENOVA, S. y VIVES, L. (2009), "Profits for passion-maximizing: Business models and value creation in creativity-driven ventures", 25 th EGOS Colloquium, Barcelona. 
Momentum Project: buscando el equilibrio entre la CReación de VAlor eConómico y VAlor SOCIAL

TYEBJEE, T. y BRUNO, A (1984), "A model of venture capitalist investment activity”, Management Science, vol. 30, núm. 9, pp. 1051-1066.

VERNIS, A. (2009), "Innovación social local a través del mercado en las organizaciones de la sociedad civil en Iberoamérica", Revista Española del Tercer Sector, núm. 13, pp. 99-134.

VERNIS, A. y IGLESIAS, M. (2010), Empresas que inspiran futuro. Ocho casos de emprendedores sociales. ESADE, Instituto de Innovación Social, Barcelona.

ZAHRA, S. A. GEDAJLOVIC, E. et al. (2009), "A typology of social entrepreneurs: Motives, search processes and ethical challenges", Journal of Business Venturing, vol. 24, núm. 5, pp. 519-532. 